\title{
Portal Cavernoma Cholangiopathy in Children and the Management Dilemmas
}

\author{
Moinak Sen Sarma* and Aathira Ravindranath \\ Department of Pediatric Gastroenterology, Sanjay Gandhi Post Graduate Institute of Medical Sciences, Lucknow, Uttar Pradesh, \\ India
}

\begin{abstract}
Portal cavernoma cholangiopathy (PCC) is one of the most harrowing complications of extrahepatic portal venous obstruction, as it determines the long-term hepatobiliary outcome. Although symptomatic PCC is rare in children, asymptomatic PCC is as common as that in adults. However, there are major gaps in the literature with regard to the best imaging strategy and management modality in children. Moreover, natural history of PCC and effect of portosystemic shunt surgeries in children are unclear. Neglected PCC would lead to difficult or recalcitrant biliary strictures that will require endoscopic therapy or bilioenteric anastomosis, both of which are challenging in the presence of extensive collaterals. There are limited studies on the effect of portosystemic shunt surgeries on the outcome of PCC in children compared to adults. In this review, we aimed to collate all existing literature on PCC in childhood and also compare with adult studies. We highlight the difficulties of this disease to provide a comprehensive platform to foster further research on PCC exclusively in children.
\end{abstract}

Citation of this article: Sen Sarma M, Ravindranath A. Portal cavernoma cholangiopathy in children and the management dilemmas. J Clin Transl Hepatol 2020;8(1):61-68. doi: 10.14218/JCTH.2019.00041.

\section{Introduction}

Extrahepatic portal venous obstruction (EHPVO) is a disorder that occurs predominantly in children and its manifestations persist throughout childhood, adolescence and into adulthood. Portal cavernoma cholangiopathy (PCC) is a distinct biliary complication of EHPVO. Cholangiography confirms hepatobiliary structural changes, whereas vascular imaging delineates venous anatomy of these collaterals. Despite EHPVO being a noncirrhotic disease with a favorable outcome, cholangiopathy is identified only when the patient becomes symptomatic. Existing literature and consensus

Keywords: Extra-hepatic portal vein obstruction; Portal cavernoma cholangiopathy; Portosystemic shunt.

Abbreviations: CBD, common bile duct; EHPVO, extrahepatic portal venous obstruction; ERC, endoscopic retrograde cholangiogram; EUS, endoscopic ultrasound; MRC, magnetic resonance cholangiogram; MRPV, magnetic resonance portovenogram; PCC, portal cavernoma cholangiopathy; PV, portal vein; SMV, superior mesenteric vein.

Received: 2 September 2019; Revised: 8 December 2019; Accepted: 1 January 2020

*Correspondence to: Moinak Sen Sarma, Department of Pediatric Gastroenterology, Sanjay Gandhi Post Graduate Institute of Medical Sciences, Lucknow, Uttar Pradesh, India. Tel: +91- 522-2495379, E-mail: moinaksen@yahoo.com statements are centered around adults with this disease; however, the problem is less highlighted in children due to paucity of literature. In this review, we attempt to outline the epidemiology of PCC, pathogenesis, symptoms, yield of investigations and management in children.

\section{Epidemiological burden of PCC}

Though it has been more than 5 decades since the first description of biliary changes in EHPVO, it still remains as one of the most dreaded complications of EHPVO, with a direct impact on prognosis. PCC is the biliary complication which encompasses all abnormalities in the extrahepatic biliary ducts, cystic duct, gall bladder and up to second generation intrahepatic biliary ducts in patients with EHPVO. ${ }^{1}$ Biliary changes are also described in patients with cirrhosis and noncirrhotic portal hypertension, but the majority of cases have been described in EHPVO. ${ }^{2}$ In adults with EHPVO, PCC is present in $90-100 \%$, of whom symptoms related to PCC are variably seen in $5-38 \% .^{3,4}$ In contrast to the West, EHPVO is the most common cause of portal hypertension in Asian children (68-76\%). ${ }^{5}$ The reported overall prevalence of PCC is $92 \%$ ( $85 \%$ asymptomatic; $7 \%$ symptomatic) in the authors' study where 72 children were screened with multimodal investigations. Initial symptoms include cholestatic jaundice and recurrent cholangitis. ${ }^{6}$ In case of persistent obstruction to biliary drainage, secondary biliary cirrhosis can occur in the long run. PCC will affect long-term hepatobiliary outcome independent of the other complications of portal hypertension in EHPVO. PCC is a silently progressive disease, seemingly innocuous yet deceptive in children.

\section{Pathogenesis}

Deciphering the natural history of PCC and predicting response to different types of surgical shunts require a thorough understanding of pathogenesis, for which it is essential to simplify the complex biliary system venous drainage. The common bile duct (CBD) is essentially drained by tributaries of the portal vein (PV) and superior mesenteric vein (SMV). It is possible that in reality the SMV drains a larger part of CBD than the PV. ${ }^{7}$ The reticulate epicholedochal and paracholedochal venous plexuses wrap around the CBD like a sieve, flush with the serosal surface. Perforator veins connect the two surface plexuses with the subepithelial veins and intramural plexuses. The surface plexuses form the marginal veins at 3 o'clock and 9 o'clock positions. Inferiorly, the veins communicate with the gastric veins, gastrocolic trunk and posterior superior pancreaticoduodenal veins. Superiorly, 
they drain into the hilar venous plexus and ultimately into the PV radicals. ${ }^{8}$

It is important to note that the posterior superior pancreaticoduodenal vein communicates with the posterior inferior pancreaticoduodenal vein and first jejunal vein, which are tributaries of SMV. Similarly, the gastrocolic trunk is also a tributary of SMV (Fig. 1). It is now possible to fathom the consequences of portal venous thrombosis. The paracholedochal and epicholedochal venous channels engorge when PV is blocked (Fig. 2). At this point, the congested bile duct drains through the SMV territory preferentially. Multiple collaterals reach out from the PV zone to the SMV, and a portal cavernoma is thus seen on imaging. However, the bypass drainage is insufficient and suboptimal, and consequently the enlarged paracholedochal veins will cause extrinsic compression on the bile ducts and the epicholedochal veins will cause fine irregularities of the bile duct contour. The subepithelial veins also enlarge to form intracholedochal varices which protrude into the bile duct lumen. ${ }^{9}$

Compression by the enlarged venous plexus was questioned to be the sole mechanism behind PCC, as portosystemic shunt surgeries failed to reverse all the biliary abnormalities. Therefore, the ischemic hypothesis was proposed, according to which ischemia to bile ducts could have occurred at the time of PV thrombosis or due to extension of thrombosis into the draining veins or the arterial network. ${ }^{10}$ With increasing duration of EHPVO, the periportal collaterals will develop surrounding fibrous connective tissue, causing a tumorous fibrous cavernoma encasing the CBD and leading to clinical consequences. It is already known that PCC will develop due to compression of the bile duct by the engorged peribiliary vessels and also due to ischemia. New issues in pathogenesis are related to the flow dynamics in the peribiliary vessels based on the extent of PV thrombosis and patency of SMV that can have long-term implications, even after portosystemic decompression. Concomitant thrombosis of the SMV occurs in as high as $64 \%$ of patients with EHPVO. ${ }^{6}$ This results in a "double-choking" effect. Neither PV nor SMV have the scope of adequate drainage of bile duct. Thus, the PCC will be far more severe when there is an associated SMV block, as shown in the study by the authors ${ }^{6}$ (Fig. 3 ).

Walser et al. ${ }^{11}$ compared 19 patients with PCC and 41 patients without PCC in PV thrombosis. They postulated that in the event of PV thrombosis, there is a preferential decompression into the gastroesophageal varices rather than peripancreatic venous structures, possibly representing a natural

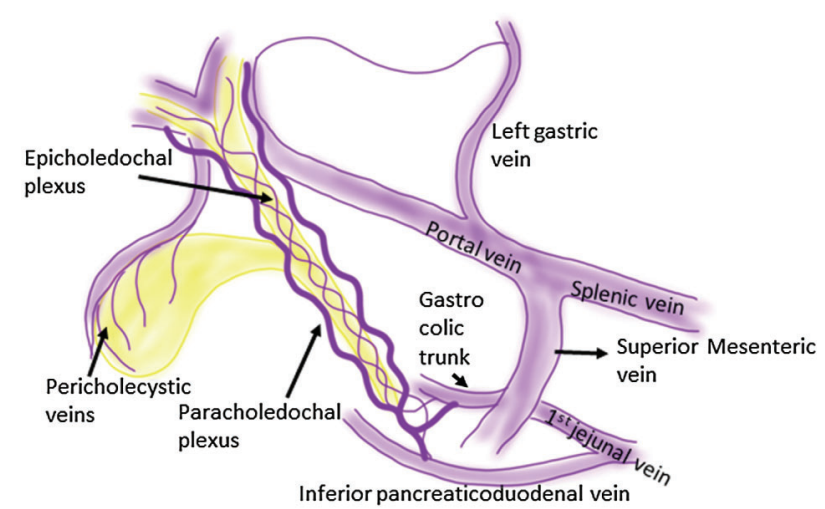

Fig. 1. Normal venous drainage of the biliary apparatus.

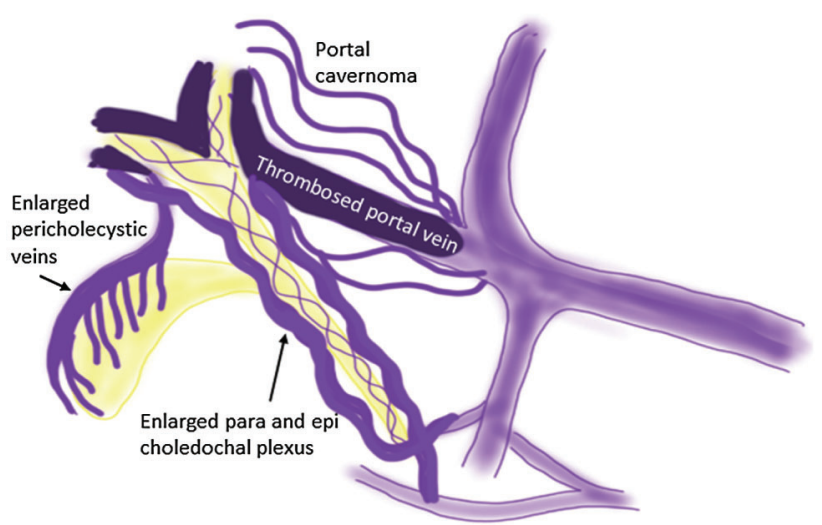

Fig. 2. Enlargement of peribiliary collaterals and development of cholangiopathy after portal vein thrombosis.

protective mechanism to avoid PCC. The authors observed that those with PCC had lower esophageal varices, smaller coronary vein diameter and acute angulation of CBD (due to compression of pancreaticoduodenal veins). Those without PCC had dilated coronary veins due to decrease in hepatopetal flow. In concomitant SMV block, collaterals may flow retrograde through the right colic and gastroepiploic veins and gastrocolic trunk. These drain into the anterior pancreaticoduodenal vein that communicates with the posterior pancreaticoduodenal vein. ${ }^{11}$ Hence, in a portomesenteric occlusion there is a preferential peribiliary flow rather than gastroesophageal flow that worsens the PCC.

\section{Implications of symptoms in children}

Though PCC has been documented in the majority of patients with EHPVO, most of the cases are asymptomatic in adults as well as in children. Adults with PCC can present with symptoms in as high as $38 \%$, but in children symptomatic PCC is seen in only $7 \%$ cases. ${ }^{4,6}$ Studies in adults have demonstrated that the probability of symptomatic PCC is related to the duration of EHPVO and, hence, it is comprehensible that the majority of the children may not present with symptoms of cholestasis as the total duration of EHPVO is shorter compared to that in adults. ${ }^{12,13}$

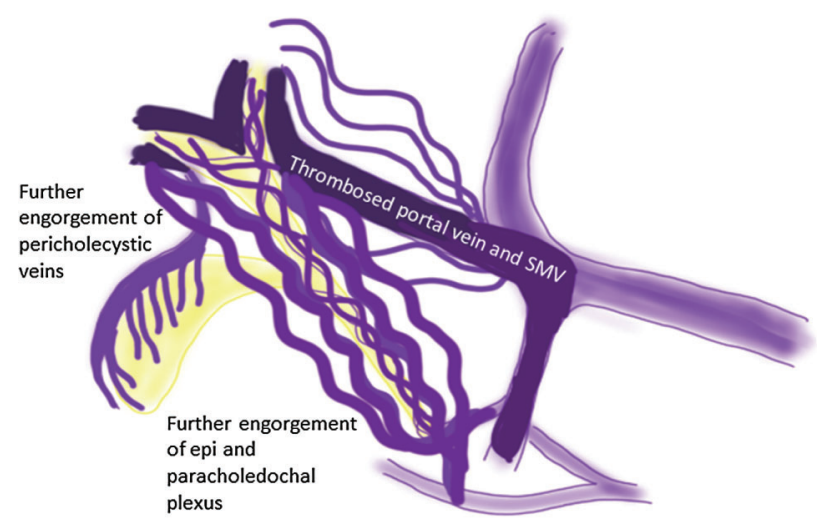

Fig. 3. Further enlargement of peribiliary collaterals when superior mesenteric vein is also thrombosed. 
In a study of adults with symptomatic PCC, the median age of presentation with symptoms of PCC was 41 years. ${ }^{14}$ The mean interval between the first presentation with variceal bleed and jaundice was 7.4 years in another study of adults. ${ }^{15}$ In a study of adults by Llop et al., it was shown that the 5-year and 10-year actuarial probability of developing symptomatic PCC after diagnosis of chronic PV thrombosis was $9 \%$ and $13 \%$, respectively. ${ }^{16}$ In the pediatric study by the authors, the age at presentation and the duration of disease in asymptomatic PCC were $13.9 \pm 2.3$ and $6.9 \pm 4.0$ years, respectively. This was significantly lower than the symptomatic group, where age and duration were $16.1 \pm 0.9$ and $11.0 \pm 1.4$ years, respectively. Age at presentation and duration of disease had significant linear correlation. ${ }^{6}$

Zargar et al. ${ }^{17}$ followed 69 EHPVO children for 15 years, and $4 \%$ developed biliary obstruction. In one of the earlier studies involving children exclusively, cholestasis was found in $6.6 \% .^{18}$ Symptoms arise due to obstruction of bile flow and result in cholestatic jaundice, pruritus, cholangitis, and gall stones. The implication of finding symptomatic PCC in children is grave. This would possibly mean tenacious strictures or stones that would entail multiple therapeutic endoscopies. A series of complications is anticipated. Endoscopic biliary intervention has technical limitations in younger children. Biliary drainage is associated with a risk of hemobilia from rupture of intracholedochal varices. Blood in the biliary tract may in turn lead to biliary sepsis, cholangiolytic abscess, and biliary stones. Endoscopic intervention is easier for lower biliary strictures than higher strictures, more so in children. Refractory strictures may necessitate bilioenteric anastomosis.

Long-standing disease results in secondary biliary cirrhosis. Secondary biliary cirrhosis is an unfortunate consequence of a problem where a primary liver disease never existed in the first place. It ultimately leads to end-stage liver disease. Considering the longevity of a child, quality of life in the growing years and gainful living, it is imperative to actively search for asymptomatic biliary changes with serial imaging. Presentation as symptomatic PCC would be akin to "missing the boat" or practically end-stage disease.

\section{Investigative yield}

Since symptomatic PCC is rare the diagnosis relies heavily on investigations. In adult studies, it has been shown that $50 \%$ of those with PCC had normal serum alkaline phosphatase and normal bilirubin at the time of evaluation, despite having jaundice in the past. ${ }^{15}$ Pediatric studies have also shown that liver biochemical tests do not differentiate between those with and without PCC. ${ }^{6}$ Thus, normal liver biochemistry does not rule out PCC. The definition of PCC itself involves demonstration of biliary abnormalities by imaging. Pertinent to children, choosing the appropriate imaging modality and defining the biliary abnormalities are imprecise. Ultrasonography is the initial screening tool and when combined with color Doppler forms a handy investigative modality for diagnosing PCC. Color Doppler discerns periportal collaterals, tortuous collateral vessels in the gall bladder, and thickened bile duct walls due to mural varices (also known as pseudocholangiocarcinoma sign). ${ }^{19}$ Though ultrasonography can detect stones, CBD dilatation and dilatation of intrahepatic biliary radicals, the exact number and levels of narrowing cannot be distinguished. Moreover, visualization of $C B D$ will be more challenging in the presence of high level echoes at the porta and bowel gas obscuration, and is operator dependent.
In the authors' study, only $29 \%$ of EHPVO had dilated intrahepatic biliary radicals on ultrasound, while $92 \%$ actually had PCC on cholangiography. ${ }^{6}$ In another study that included adults and children with EHPVO, ultrasound detected biliary abnormalities in 54\% that included intrahepatic biliary radicals dilatation, $\mathrm{CBD}$ narrowing, $\mathrm{CBD}$ wall thickening due to mural varices, and choledocholithiasis. ${ }^{20}$ Despite the advantages of absence of irradiation and no requirement for sedation, ultrasonography is a poor screening tool for PCC. Computed tomography of the abdomen can clearly demonstrate portal cavernoma, peribiliary varices, parabiliary varices, extent of portal thrombosis, and other collaterals. Maximum information is provided by the portal venous phase. Computed tomography can reveal bile duct narrowing and also show the corresponding compression by peribiliary varices. ${ }^{21}$ The use of computed tomography in children has the disadvantage of exposure to radiation, especially with repeated imaging in the growing years.

\section{Defining biliary and vascular changes by magnetic resonance imaging}

Combination of magnetic resonance cholangiogram (MRC) and magnetic resonance portovenogram (MRPV) provides the best anatomical details. MRC with heavily T2-weighted images and maximum intensity projection can create excellent reconstruction of the biliary tree, similar to the images obtained by direct cholangiography. ${ }^{22}$ There are two major classification systems of biliary changes on cholangiography described in adult patients. The Chandra's classification is based on the anatomy of the biliary changes (type I: involvement of extrahepatic bile duct; type II: involvement of intrahepatic bile ducts only; type IIIa: involvement of extrahepatic and unilateral intrahepatic bile ducts; type IIIb: involvement of extrahepatic and bilateral intrahepatic bile ducts). ${ }^{2}$ However, this classification does not reflect the severity. The Llop's classification grades PCC as per the severity (grade I: irregularities and angulations of biliary tree; grade II: stenosis without dilatation; grade III: strictures with upstream dilatation) (Fig. 4). Biliary dilatation was defined as $\geq 4 \mathrm{~mm}$ diameter of intrahepatic ducts and $\geq 7 \mathrm{~mm}$ diameter of extrahepatic duct. ${ }^{16}$ Only the patients with grade III PCC had symptoms. However, these bile duct diameters cannot be applied in children of all ages.

In the pediatric study by Gauthier-Villars et al., ${ }^{18}$ the intrahepatic biliary diameter ranged from 2 to $15 \mathrm{~mm}$ and extrahepatic biliary diameter from 3 to $11 \mathrm{~mm}$. In the authors' pediatric study, PCC was graded as per modified Llop's classification, by incorporating upper limits of biliary dilatation as reported by Zhang et al. ${ }^{6,23}$ Hence, in children, grade III strictures with dilatation is defined as upstream dilatation of CBD and common hepatic duct, with diameter $>4.1 \mathrm{~mm}$, and central intrahepatic biliary diameter $>2 \mathrm{~mm}$. Thus, interpretation of MRC findings in children should be done with the necessary modifications. Another classification used in adults based on the compressive and ischemic changes on biliary ducts divides PCC into varicoid, fibrotic and mixed types. In the varicoid type, extrinsic compressions caused by dilated peribiliary vessels produce irregular wavy contour of the bile ducts and in the fibrotic type, ischemic changes will produce bile duct narrowing with proximal dilatations. Most cases will have a mixture of both these varieties. ${ }^{24}$ None of the classifications include cholelithiasis and choledocholithiasis, which will have clinical implications. In the authors' own 

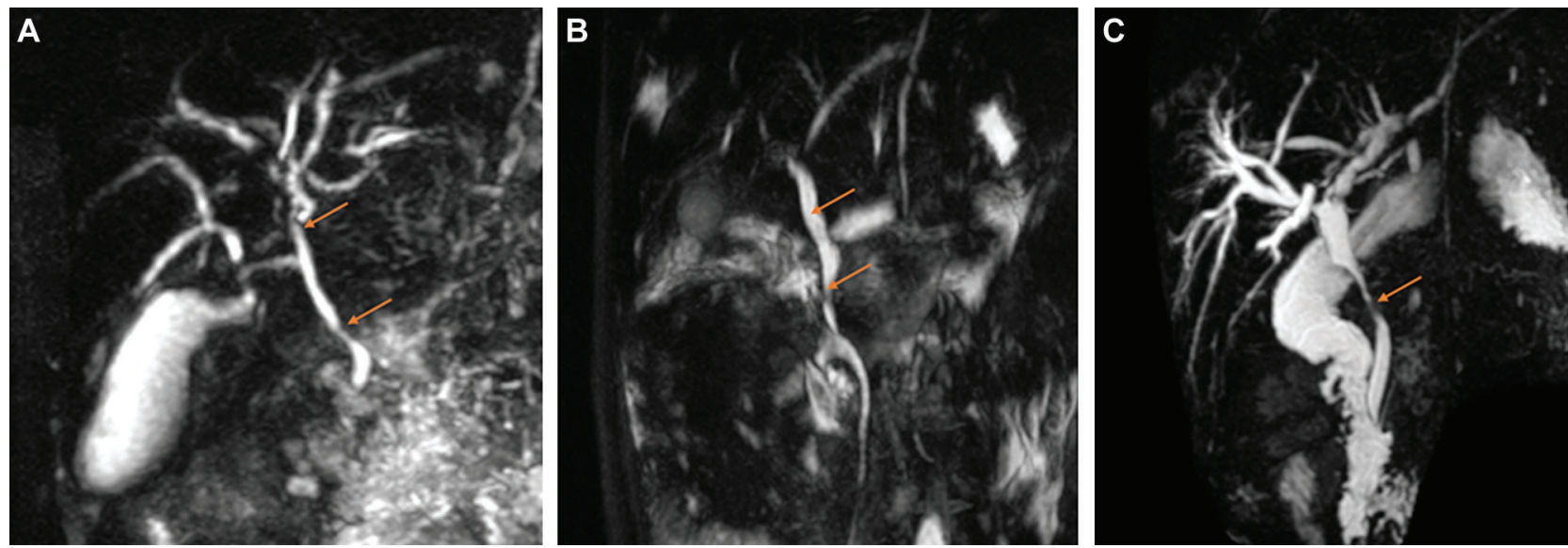

Fig. 4. Magnetic resonance cholangiogram. A: Irregularities of the biliary tree (arrow). B: Indentations on the biliary tree (arrow). C: Strictures (arrow) in biliary tree with upstream dilatation.

experience, in children, presence of biliary stones and intrahepatic strictures have a more convoluted outcome than simple extrahepatic biliary strictures in terms of therapeutic endoscopy. Hence, there is a dire need to reclassify PCC by taking into consideration the symptoms, anatomy, and grade of biliary changes on MRC.

MRPV can delineate the extent of PV thrombosis, extent of cavernoma, peribiliary and gall bladder collaterals, and distribution of all intra-abdominal collaterals. The relationship of biliary strictures with peribiliary collaterals can also be illustrated. However, there are no standard grading systems for density of peribiliary collaterals. Certain MRPV findings have prognostic significance and aid in surgical planning. A patent left branch of PV and the patency of the confluence should be keenly looked for while planning the Meso-Rex shunt. For alternate portosystemic shunts, patency of the splenic vein and SMV ought to be determined. As alluded to earlier, SMV forms an important drainage pathway for the peribiliary vessels. SMV block is associated with greater severity of PCC in children on MRC. ${ }^{6}$ Though MRC and MRPV provide crucial information, children have some unique issues, like requirement of sedation and poor breath-holding which will compromise the image quality. The authors believe that pre-emptive screening for PCC is warranted in children with EHPVO at the time of presentation.

\section{Limitation of endoscopic retrograde cholangiogram (ERC)}

The earliest of studies describing PCC used ERC for diagnosis. In the study by Dilawari et al., ${ }^{3}$ abnormalities in the CBD were seen in $90 \%$, left bile duct in $100 \%$ and right duct in $56 \%$. The various findings described by Khuroo et al. ${ }^{4}$ included caliber irregularity, ectasias, external impressions, strictures, upstream dilatation, displacement of duct, angulations, and pruning of intrahepatic ducts. The role of diagnostic ERC in the current era is limited. In children with PCC, diagnostic ERC can produce iatrogenic cholangitis and expose to radiation. Moreover, it needs expertise, and size of outer diameter of scope is a major limiting factor for younger children. Hence, diagnostic ERC is not preferred over MRC. Therapeutic ERC is indicated in cases with cholangitis, choledocholithiasis and biliary strictures which are symptomatic or persisting after portosystemic shunt surgeries. ${ }^{25}$
Role of pediatric endoscopic ultrasonography (EUS)

EUS is an attractive option for diagnosing PCC. Slimmer diagnostic endosonoscopes are now available. EUS can provide information about the vascular changes in the peribiliary area and choledochal varices. Similar to EUS use in adults, it can illustrate dilated choledochal perforators, intracholedochal, and intramural gall bladder varices in children. Children have biliary diameters that are narrower than in adults, more so due to compressive changes in PCC. Hence on EUS, the narrow bile duct surrounded by cavernoma is strenuously identified and appears as the "last log of wood engulfed in flames" (Fig. 5). In contrast to adults, the fine differentiation of epi-, para- and peri-choledochal varices, in reality, is difficult, as subepithelial and fibromuscular layers

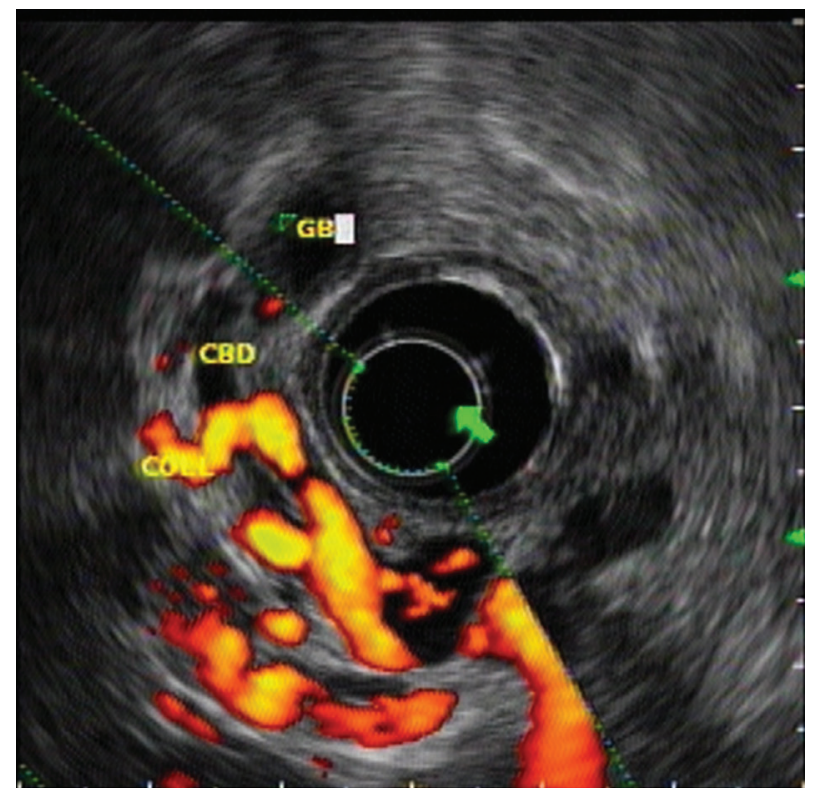

Fig. 5. Endoscopic ultrasound Doppler image showing dilated common bile duct engulfed by collaterals.

Abbreviations: GB, gall bladder; CBD, common bile duct; COLL, collaterals. 
are not adequately demonstrated in children. In the only pediatric study that incorporated EUS in PCC evaluation, it was found that there was a modest role in detecting biliary calculi at the lower end of CBD that could be missed on MRC. ${ }^{6}$

Further studies are needed to assess the role of EUS in the diagnosis and prognostication of PCC in children. EUS could hence play a vital role before ERC by providing information about the exact location of intracholedochal varices in averting hemobilia during therapeutic ERC. The major drawbacks of endoscopic ultrasonography are lack of compatible sizes of echoendoscopes for younger children and relatively poor delineation of higher biliary strictures. As such, this modality may be used as an ancilliary investigation.

\section{Dilemmas in definitive management}

PCC determines the long-term hepatobiliary outcome in children with EHPVO. ${ }^{26}$ In developing countries, late referral of EHPVO patients is additive to the management complexity. Management of PCC is a situation of major dilemma for surgeons and physicians. Choice of portosystemic shunt, adequate decompression of biliary varices, appropriate time for bilioenteric anastomosis and prophylactic biliary dilatation for strictures are well debated. Despite active screening for PCC in all children, we must understand that symptoms arise as a result of procrastination of treating asymptomatic PCC. Symptomatic PCC definitely requires biliary drainage but requirement of biliary decompression in asymptomatic PCC is a dilemma not only in children but also in adults.

After detecting PCC, the logical step forward has to be decompression of the portal hypertension with portosystemic shunt surgery. In those with symptomatic PCC, endoscopic therapy may be required before the shunt surgery. Presently it is viewed that endoscopic therapy is akin to a tight rope walk that has to balance between the risks involved and adequacy of biliary drainage. Hence, it is reserved for select cases of cholangitis and choledocholithiasis. Limitations such as lack of appropriate sized endoscopes and biliary metallic stents (not approved yet) are unique issues in children.

\section{Problems of portosystemic shunt surgeries}

Meso-Rex shunt is the most physiological shunt and the procedure of choice in EHPVO. The recessus of Rex in the left branch of PV is the part of the umbilical venous pathway that remains patent despite PV block and creation of a conduit between SMV and recessus of Rex decompresses the portal system in the most physiological manner. ${ }^{27}$ In the study by Gauthier-Villars et al., ${ }^{18}$ two of eight children with symptomatic PCC underwent Rex anastomosis and liver biochemistry normalized after the shunt surgery. However, Meso-Rex shunt remains a utopian procedure for most children with EHPVO as the left branch of PV is not patent. Despite Meso-Rex bypass being recommended in all children with EHPVO, it might not be possible in those with non-favorable vascular anatomy. In addition, in cases with long standing portal hypertension, issues related to large spleen predominate and the need for appropriate alternative portosystemic shunts then arises. Total and selective portosystemic shunts have their own demerits of encephalopathy and liver ischemia.

The effect of portosystemic shunt surgeries on PCC has been shown in small series. In the study by Dhiman et al. ${ }^{10}$ which included five children, post-portosystemic shunt ERC showed that angulations and ectasias persisted and strictures reversed partially in all, except one. This study paved way for the ischemic hypothesis. In another study of adults with EHPVO, among 19 patients with PCC, 10 underwent splenorenal shunt surgery with jugular graft, after which symptoms resolved in 7 but there was no significant improvement in liver function tests. Angiograms performed in five patients after shunt surgery showed that portal cavernoma flow decreased in four patients, all of whom had patent SMV, and remained the same in one whose SMV was blocked. ${ }^{28}$ In a recent study comprising 25 children with EHPVO who underwent central end to side splenorenal shunt (where Meso-Rex shunt was not feasible), despite the patency of central endto-side splenorenal shunt and decompression of the gastroesophageal bed, the PCC did not ameliorate at a median follow-up of 18 months. All those who had progressive PCC had concomitant SMV block ${ }^{29}$ (Fig. 6) SMV block assumes great importance, as it not only deems the case unfavorable for Meso-Rex bypass, it is also associated with greater severity of PCC in children. It is possible that an early alternative pre-emptive portosystemic shunt surgery is warranted in this group. ${ }^{6}$

The choice of portosystemic shunt surgery and the patency of SMV will play crucial roles in determining the final outcome of PCC. Summary of all studies on PCC in
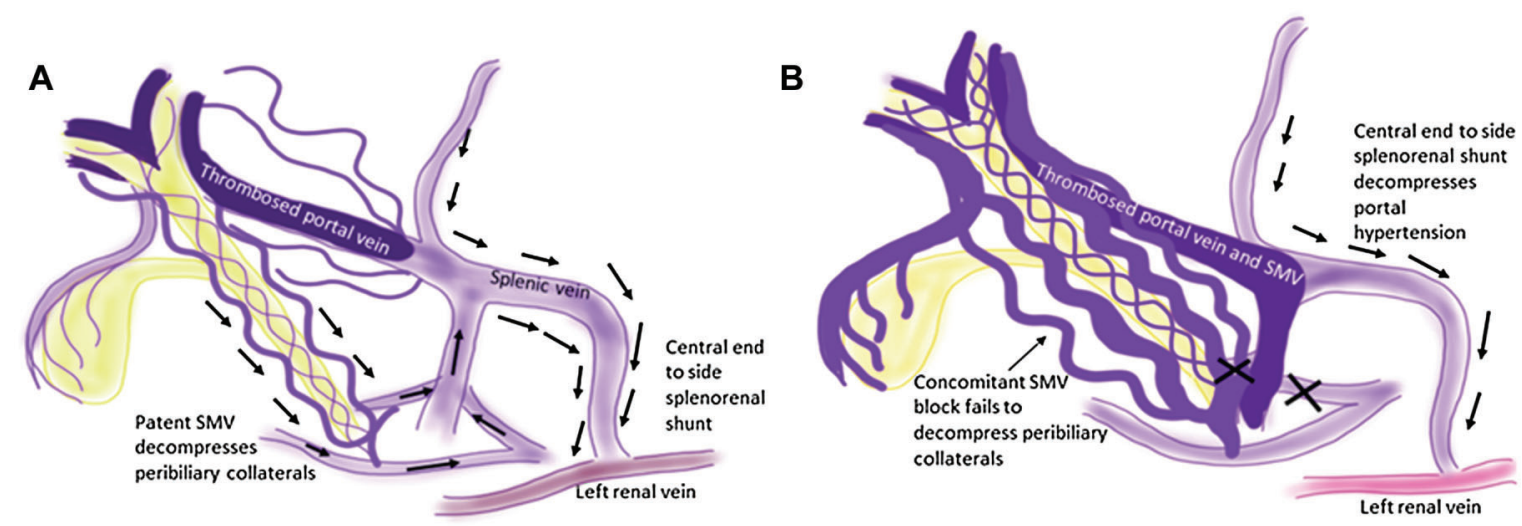

Fig. 6. Peribiliary collaterals. A: Decompressed after splenorenal shunt when SMV is patent. B: Increased after splenorenal shunt when SMV is blocked. Abbreviation: SMV, superior mesenteric vein. 
Sen Sarma M. et al: Portal cavernoma cholangiopathy in children

Table 1. Summary of the studies on portal cavernoma cholangiopathy in children

\begin{tabular}{|c|c|c|c|c|c|c|}
\hline $\begin{array}{l}\text { Author/Country/ } \\
\text { Year/Sample } \\
\text { size }\end{array}$ & Incidence & $\begin{array}{l}\text { Median } \\
\text { age in } \\
\text { years }\end{array}$ & $\begin{array}{l}\text { Duration of } \\
\text { disease in } \\
\text { years }\end{array}$ & Symptoms & Treatment & Outcome \\
\hline $\begin{array}{l}\text { Gauthier- } \\
\text { Villars }{ }^{18} / \\
\text { France/2004/ } \\
n=121\end{array}$ & $6.6 \%(8 / 121)$ & 5 & NA & $\begin{array}{l}\text { Jaundice } \\
(n=3) \\
\text { Prutitus }(n=3) \\
\text { Hepatomegaly } \\
(n=2)\end{array}$ & $\begin{array}{l}\text { Mesocaval } \\
\text { shunt }(n=5) \\
\text { Jejunocaval } \\
\text { shunt }(n=1) \\
\text { Meso-Rex } \\
\text { shunt }(n=2)\end{array}$ & $\begin{array}{l}\text { Symptom } \\
\text { resolution } \\
(n=8) \\
\text { LFT } \\
\text { normalization } \\
(n=8) \\
\text { Resolution } \\
\text { of biliary } \\
\text { dilatation } \\
(n=7)\end{array}$ \\
\hline $\begin{array}{l}\text { Zargar }^{17} / \text { India/ } \\
2004 / n=69\end{array}$ & $4.3 \%(3 / 69)$ & NA & NA & NA & $\begin{array}{l}\text { Portosystemic } \\
\text { shunt surgery } \\
\text { (details NA) }\end{array}$ & NA \\
\hline $\begin{array}{l}\text { Sen Sarma } / \\
\text { India/2018/ } \\
n=72\end{array}$ & $\begin{array}{l}92 \%(66 / 72) \\
82 \%- \\
\text { asymptomatic, } \\
7 \% \text { - symptomatic }\end{array}$ & 13.9 & 6.8 & $\begin{array}{l}\text { Jaundice } \\
(n=3) \\
\text { Cholangitis } \\
(n=2)\end{array}$ & NA & NA \\
\hline $\begin{array}{l}\text { Ravindranath }{ }^{29} / \\
\text { India/ } \\
2019 / n=25\end{array}$ & NA & 16 & 6.4 & $\begin{array}{l}\text { Cholangitis } \\
(n=1) \\
\text { Jaundice } \\
(n=1)\end{array}$ & $\begin{array}{l}\text { Central end-to- } \\
\text { side } \\
\text { splenorenal } \\
\text { shunt }(n=24) \\
\text { Mesocaval } \\
\text { shunt } \\
(n=1)\end{array}$ & $\begin{array}{l}\text { Symptom } \\
\text { resolution } \\
(n=2) \\
\text { Resolution } \\
\text { of biliary } \\
\text { dilatation } \\
(n=0)\end{array}$ \\
\hline
\end{tabular}

Abbreviations: LFT, liver function test; NA, not available.

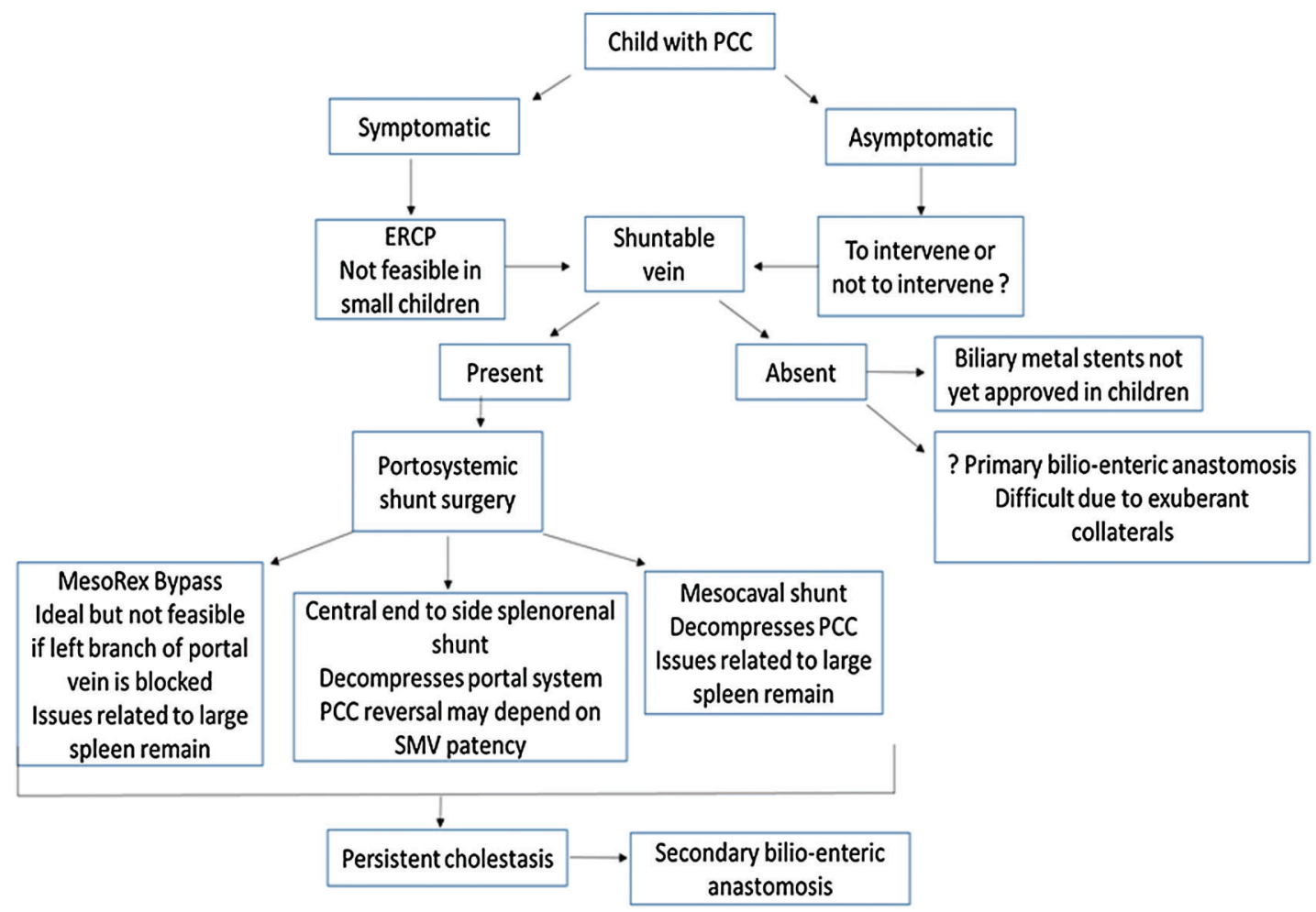

Fig. 7. Summary of management dilemmas of portal cavernoma cholangiopathy in children.

Abbreviations: ERCP, endoscopic retrograde cholangiopancreatography; PCC, portal cavernoma cholangiopathy; SMV, superior mesenteric vein. 
children is provided in Table 1. Portosystemic shunt surgery is not the final answer to PCC as it may not resolve in all. In those in whom biliary strictures persist, a second stage bilioenteric anastomoses may be contemplated. ${ }^{30}$ Bilioenteric anastomosis is surgically daunting in naive EHPVO patients, due to dense hepatobiliary collaterals. The therapeutic strategy in asymptomatic PCC is more perplexing, as complete reversal after portosystemic shunt surgery is still uncertain (Fig. 7). Although symptomatic PCC occurs more often in adults, masterly inactivity in children with asymptomatic PCC would increase the burden of complicated PCC and non-shuntable anatomy as they enter adulthood. Future studies are required to address the optimum management modality in asymptomatic PCC.

\section{Liver transplantation for PCC}

After failure of endoscopic and surgical treatment for PCC the only other feasible option is liver transplantation, though the available literature is scarce. The indications of liver transplantation in this scenario would be recurrent cholangitis, secondary biliary cirrhosis, or intrahepatic cavernoma. ${ }^{31,32}$ The unique surgical challenge in those with PCC would be to achieve good portal inflow to the graft by using grafts from other veins or anastomosis with large recipient collaterals. $^{33,34}$ Thus, deceased donor liver transplantation is preferred over living donor due to the availability of suitable graft PV length and also other venous conduits. ${ }^{35}$

\section{Conclusions}

PCC is an important complication of EHPVO that affects the long-term prognosis in EHPVO. Optimum modality of imaging and management of asymptomatic PCC in children still has many unanswered questions. Large scale studies are needed to discern the natural history of PCC in children and the effect of different types of portosystemic shunt surgeries on PCC with respect to SMV patency.

\section{Funding}

None to declare.

\section{Conflict of interest}

The authors have no conflict of interests related to this publication.

\section{Author contributions}

Conceptualized the article and supervised manuscript writing (MSS), collected data and drafted the manuscript (AR), approved the final manuscript as submitted and agree to be accountable for all aspects of the work (MSS, AR).

\section{References}

[1] Chawla Y, Agrawal S. Portal cavernoma cholangiopathy - history, definition and nomenclature. J Clin Exp Hepatol 2014;4:S15-S17. doi: 10.1016/j.jceh. 2013.04.001.

[2] Chandra R, Kapoor D, Tharakan A, Chaudhary A, Sarin SK. Portal biliopathy. J Gastroenterol Hepatol 2001;16:1086-1092. doi: 10.1046/j.1440-1746. 2001.02562.x.
[3] Dilawari JB, Chawla YK. Pseudosclerosing cholangitis in extrahepatic portal venous obstruction. Gut 1992;33:272-276. doi: 10.1136/gut.33.2.272.

[4] Khuroo MS, Yattoo GN, Zargar SA, Javid G, Dar MY, Khan BA, et al. Biliary abnormalities associated with extrahepatic portal venous obstruction. Hepatology 1993;17:807-813. doi: 10.1002/hep.1840170510.

[5] Yachha SK. Portal hypertension in children: an Indian perspective. J Gastroenterol Hepatol 2002;17 Suppl 3:S228-S231. doi: 10.1046/j.1440-1746. 17.s3.5.x.

[6] Sen Sarma M, Yachha SK, Rai P, Neyaz Z, Srivastava A, Poddar U. Cholangiopathy in children with extrahepatic portal venous obstruction. J Hepatobiliary Pancreat Sci 2018;25:440-447. doi: 10.1002/jhbp.582.

[7] Sen Sarma M, Yachha SK, Chaudhary A. Portal biliopathy. In: Barreto SG, Windsor JA. Surgical diseases of the pancreas and biliary tree. Singapore: Springer; 2018:209-218.

[8] Ramesh Babu CS, Sharma M. Biliary tract anatomy and its relationship with venous drainage. J Clin Exp Hepatol 2014;4:S18-S26. doi: 10.1016/j.jceh. 2013.05.002.

[9] Sharma M, Pathak A. Perforators of common bile duct wall in portal hypertensive biliopathy (with videos). Gastrointest Endosc 2009;70:1041-1043. doi: $10.1016 /$ j.gie.2009.03.040.

[10] Dhiman RK, Puri P, Chawla Y, Minz M, Bapuraj JR, Gupta S, et al. Biliary changes in extrahepatic portal venous obstruction: compression by collaterals or ischemic? Gastrointest Endosc 1999;50:646-652. doi: 10. 1016/s0016-5107(99)80013-3.

[11] Walser EM, Runyan BR, Heckman MG, Bridges MD, Willingham DL, PazFumagalli $\mathrm{R}$, et al. Extrahepatic portal biliopathy: proposed etiology on the basis of anatomic and clinical features. Radiology 2011;258:146-153. doi: 10.1148/radiol.10090923.

[12] Peter L, Dadhich SK, Yachha SK. Clinical and laboratory differentiation of cirrhosis and extrahepatic portal venous obstruction in children. J Gastroenterol Hepatol 2003;18:185-189. doi: 10.1046/j.1440-1746.2003.02943.x.

[13] Shukla A, Gupte A, Karvir V, Dhore P, Bhatia S. Long term outcomes of patients with significant biliary obstruction due to portal cavernoma cholangiopathy and extra-hepatic portal vein obstruction (EHPVO) with no shuntable veins. J Clin Exp Hepatol 2017;7:328-333. doi: 10.1016/j.jceh.2017.04.003.

[14] Oo YH, Olliff S, Haydon G, Thorburn D. Symptomatic portal biliopathy: a single centre experience from the UK. Eur J Gastroenterol Hepatol 2009; 21:206-213. doi: 10.1097/MEG.0b013e3283060ee8.

[15] Khare R, Sikora SS, Srikanth G, Choudhuri G, Saraswat VA, Kumar A, et al. Extrahepatic portal venous obstruction and obstructive jaundice: approach to management. J Gastroenterol Hepatol 2005;20:56-61. doi: 10.1111/j. 1440-1746.2004.03528.x.

[16] Llop E, de Juan C, Seijo S, García-Criado A, Abraldes JG, Bosch J, et al. Portal cholangiopathy: radiological classification and natural history. Gut 2011;60: 853-860. doi: 10.1136/gut.2010.230201.

[17] Zargar SA, Yattoo GN, Javid G, Khan BA, Shah AH, Shah NA, et al. Fifteenyear follow up of endoscopic injection sclerotherapy in children with extrahepatic portal venous obstruction. J Gastroenterol Hepatol 2004;19: 139-145. doi: 10.1111/j.1440-1746.2004.03224.x.

[18] Gauthier-Villars M, Franchi S, Gauthier F, Fabre M, Pariente D, Bernard O. Cholestasis in children with portal vein obstruction. J Pediatr 2005;146:568573. doi: 10.1016/j.jpeds.2004.12.025.

[19] Denys A, Hélénon O, Lafortune M, Corréas JM, Patriquin H, Moreau JF, et al. Thickening of the wall of the bile duct due to intramural collaterals in three patients with portal vein thrombosis. AJR Am J Roentgenol 1998;171:455456. doi: 10.2214/ajr.171.2.9694474.

[20] Kessler A, Graif M, Konikoff F, Mercer D, Oren R, Carmiel M, et al. Vascular and biliary abnormalities mimicking cholangiocarcinoma in patients with cavernous transformation of the portal vein: role of color Doppler sonography. J Ultrasound Med 2007;26:1089-1095. doi: 10.7863/jum.2007.26.8.1089.

[21] Aguirre DA, Farhadi FA, Rattansingh A, Jhaveri KS. Portal biliopathy: imaging manifestations on multidetector computed tomography and magnetic resonance imaging. Clin Imaging 2012;36:126-134. doi: 10.1016/j.clinimag. 2011.07.001.

[22] Condat B, Vilgrain V, Asselah T, O'Toole D, Rufat P, Zappa M, et al. Portal cavernoma-associated cholangiopathy: a clinical and MR cholangiography coupled with MR portography imaging study. Hepatology 2003;37:13021308. doi: $10.1053 /$ jhep.2003.50232.

[23] Zhang $Y$, Wang XL, Li SX, Bai YZ, Ren WD, Xie LM, et al. Ultrasonographic dimensions of the common bile duct in Chinese children: results of 343 cases. J Pediatr Surg 2013;48:1892-1896. doi: 10.1016/j.jpedsurg.2012.11.047.

[24] Shin SM, Kim S, Lee JW, Kim CW, Lee TH, Lee SH, et al. Biliary abnormalities associated with portal biliopathy: evaluation on MR cholangiography. AJR Am J Roentgenol 2007;188:W341-W347. doi: 10.2214/AJR.05.1649.

[25] Bhatia V. Endoscopic retrograde cholangiography in portal cavernoma cholangiopathy - results from different studies and proposal for uniform terminology. J Clin Exp Hepatol 2014;4:S37-S43. doi: 10.1016/j.jceh.2013.05. 013.

[26] Kumar M, Saraswat VA. Natural history of portal cavernoma cholangiopathy. J Clin Exp Hepatol 2014;4:S62-S66. doi: 10.1016/j.jceh.2013.08.003. 
Sen Sarma M. et al: Portal cavernoma cholangiopathy in children

[27] Shneider BL, de Ville de Goyet J, Leung DH, Srivastava A, Ling SC, Duché M, et al. Primary prophylaxis of variceal bleeding in children and the role of MesoRex Bypass: Summary of the Baveno VI Pediatric Satellite Symposium. Hepatology 2016;63:1368-1380. doi: 10.1002/hep.28153.

[28] Vibert E, Azoulay D, Aloia T, Pascal G, Veilhan LA, Adam R, et al. Therapeutic strategies in symptomatic portal biliopathy. Ann Surg 2007;246:97-104. doi: 10.1097/SLA.0b013e318070cada.

[29] Ravindranath A, Sen Sarma M, Yachha SK, Lal R, Singh S, Srivastava A, et al. Outcome of portosystemic shunt surgery on pre-existing cholangiopathy in children with extrahepatic portal vein obstruction. J Hepatobiliary Pancreat Sci 2019. doi: 10.1002/jhbp.692.

[30] Chaudhary A, Dhar P, Sarin SK, Sachdev A, Agarwal AK, Vij JC, et al. Bile duct obstruction due to portal biliopathy in extrahepatic portal hypertension: surgical management. Br J Surg 1998;85:326-329. doi: 10.1046/j.1365-2168. 1998.00591.x.
[31] Dhiman RK, Saraswat VA, Valla DC, Chawla Y, Behera A, Varma V, et al. Portal cavernoma cholangiopathy: consensus statement of a working party of the Indian national association for study of the liver. J Clin Exp Hepatol 2014;4: S2-S14. doi: 10.1016/j.jceh.2014.02.003.

[32] Hajdu CH, Murakami T, Diflo T, Taouli B, Laser J, Teperman L, et al. Intrahepatic portal cavernoma as an indication for liver transplantation. Liver Transpl 2007;13:1312-1316. doi: 10.1002/lt.21243.

[33] Filipponi F, Urbani L, Catalano G, Iaria G, Biancofiore G, Cioni R, et al. Portal biliopathy treated by liver transplantation. Transplantation 2004;77:326327. doi: 10.1097/01.TP.0000101795.29250.10.

[34] Zhang M, Guo C, Pu C, Ren Z, Li Y, Kang Q, et al. Adult to pediatric living donor liver transplantation for portal cavernoma. Hepatol Res 2009;39:888897. doi: 10.1111/j.1872-034X.2009.00526.x.

[35] Gupta S, Taneja S. Liver transplantation for portal cavernoma cholangiopathy. J Clin Exp Hepatol 2014;4:S85-S87. doi: 10.1016/j.jceh.2014.01.001. 